\title{
ASSOCIAÇÃO ENTRE TONTURAS, QUEDASE TESTE DO ALCANCE FUNCIONAL EM IDOSOS DA COMUNIDADE
}

\author{
Adriane Ribeiro Teixeira ${ }^{1}$ \\ Andréa Kruger Gonçalves² \\ Cíntia de la Rocha Freitas ${ }^{3}$ \\ Ana Maria Pujol Vieira dos Santos ${ }^{4}$ \\ Deborah Salle Levy ${ }^{5}$ \\ Maira Rozenfeld Olchik ${ }^{6}$ \\ Sílvia Dornelles ${ }^{7}$ \\ Ângelo José Gonçalves Bós ${ }^{8}$
}

\footnotetext{
1 Fonoaudióloga, Professora da Universidade Federal do Rio Grande do Sul, Departamento de Psicologia do Desenvolvimento e da Personalidade - Instituto de Psicologia. E-mail: adriane.teixeira@ufrgs.br 2 Educadora Física - Professora da Universidade Federal do Rio Grande do Sul, Departamento de Educação Física. E-mail: andreakg@terra.com.br

3 Educadora Física - Professora da Universidade Federal de Santa Catarina. E-mail: cintiadelarocha@ gmail.com

4 Bióloga, Professora da Universidade Luterana do Brasil. E-mail: anamariapujo|@gmail.com

5 Fonoaudióloga, Professora da Universidade Federal do Rio Grande do Sul, Departamento de Psicologia do Desenvolvimento e da Personalidade - Instituto de Psicologia. E-mail: deborahslevy@terra. com.br

6 Fonoaudióloga, Professora da Universidade Federal do Rio Grande do Sul, Departamento de Cirurgia e Ortopedia - Faculdade de Odontologia. E-mail: mairarozenfeld@hotmail.com

7 Fonoaudióloga, Professora da Universidade Federal do Rio Grande do Sul, Departamento de Psicologia do Desenvolvimento e da Personalidade - Instituto de Psicologia. E-mail: dornella@gmail.com

8 Médico, Professor do Programa de Pós-Graduação em Gerontologia Biomédica da Pontifícia Universidade Católica do Rio Grande do Sul. E-mail: angelo.bos@pucrs.br
} 
resumo

Este estudo teve como objetivo verificar a associação entre a tontura, o teste do alcance funcional e o histórico de quedas. Foram avaliados 50 idosos, utilizando-se entrevista elaborada para este estudo e o teste de alcance funcional. Os resultados evidenciaram que 52\% dos idosos apresentavam tontura e 24\% relataram queda nos últimos 6 meses. No teste do alcance funcional 38\% dos idosos atingiram índices superiores a $25 \mathrm{~cm}$. A análise estatística foi feita utilizando-se os testes Qui-Quadrado, Mann-Whitney e Kruskal-Wallis e demonstrou que não houve associação entre as variáveis pesquisadas.

palavras-chave

Tontura. Quedas. Idoso. Equilíbrio Postural.

\section{Introdução}

Quedas são deslocamentos não intencionais do corpo para um nível inferior à posição inicial com incapacidade de correção em tempo hábil, determinados por circunstâncias multifatoriais, comprometendo a estabilidade (SOCIEDADE BRASILEIRA DE GERIATRIA E GERONTOLOGIA, 2008; GUIMARÃES et al., 2004).

As quedas são um problema de saúde pública entre os idosos, considerando-se a mortalidade, a morbidade e os custos sociais e econômicos delas decorrentes (GUIMARÃES; FARINATTI, 2005). Além disso, sabe-se que as quedas são importantes fatores causais de aumento no nível de dependência do idoso, pois afetam sua capacidade funcional (MACHADO et al., 2009), determinando um alto custo pessoal e organizacional (SNOOKS et al., 2010).

Segundo Perracini e Ramos (2002), aproximadamente 30\% dos idosos em países ocidentais sofrem quedas pelo menos uma vez por ano e cerca de $50 \%$ têm duas, ou mais quedas. Estima-se que 50\% das quedas originem algum tipo de lesão (MOTTA et al., 2010). Além das fraturas e do risco de morte, cair gera restrição nas atividades, declínio na saúde, risco de hospitalização e posterior institucionalização. Para Tinetti et al. (2000) e Fabrício, Rodrigues e Costa Jr. (2004), a queda pode ser considerada uma síndrome geriátrica, por ser um evento multifatorial e homogêneo, que decorre do efeito cumulativo dos déficits nos múltiplos sistemas, imputando aos idosos maior vulnerabilidade nas atividades diárias. Estudos realizados por Motta et al. 
(2010) confirmam a prevalência de 30\% de quedas em idosos. Lopes et al. (2010) analisaram quedas em 118 idosos que frequentavam uma clínica escola e constataram que $27,1 \%$ apresentavam histórico positivo para esse dado.

O equilíbrio corporal é dependente de vários sistemas: o proprioceptivo, o visual e o vestibular, os quais são coordenados pelo sistema nervoso central. Falhas podem se manifestar como desequilíbrio, tontura e quedas (BOTTEGA; LOBO; TEIXEIRA, 2006, BRONSTEIN; LEMPERT, 2010). Para Guimarães et al. (2004), o número de quedas em idosos ocorre devido à instabilidade postural, por alterações dos sistemas sensoriais e motores, decorrentes, ou não, do envelhecimento. O termo tontura é usado para descrever uma ampla variedade de sensações de perturbação do equilíbrio corporal e pode surgir em qualquer idade, mas é predominante em adultos e idosos (GANANÇA; CAOVILLA, 2000).

Estudo realizado por Tinetti et al. (2000) com 1087 idosos evidenciou que $24 \%$ relataram tontura. Segundo Koga, Resende e Mor (2004), aproximadamente $40 \%$ dos indivíduos com idade superior a 40 anos apresentam alterações de equilíbrio e episódios de tontura. Essa prevalência aumenta para $85 \%$ quando são analisados indivíduos com idade igual, ou superior a 65 anos (FELIPE et al., 2008) sendo explicada pelo declínio funcional do sistema vestibular, em função do envelhecimento (MANTELLO et al., 2008).

Fanelli, Barreto Filho e Raquel (2002), porém, discordam dessa afirmativa. Para esses autores, as causas da tontura em idosos podem estar direta ou indiretamente relacionadas a distúrbios do sistema vestibular, mas não podem ser atribuídas somente ao envelhecimento.

A tontura pode provocar medo de realizar movimentos de cabeça, de lugares altos, de olhar para baixo e gerar restrição de atividades sociais, familiares e profissionais, além de aumentar o risco de quedas (FANELLI; BARRETO FILHO; RAQUEL, 2002, TINETTI et al., 2000). Pesquisa feita por Gazzola et al. (2006) evidenciou que $25 \%$ das quedas em um grupo de 64 idosos com vestibulopatia crônica foram causadas por tontura.

A tontura também pode estar relacionada a sintomas emocionais e psicológicos, tais como ansiedade, pânico, depressão, preocupações excessivas com a saúde e medo de executar as atividades que possam provocar a tontura e as quedas (GAZZOLA et al., 2009, PATATAS; GANANÇA; GANANÇA, 2009).

Outros fatores que podem levar os idosos a sofrer quedas são as alterações de força e flexibilidade. Com o objetivo de identificar alterações dinâmicas do controle postural, Duncan et al. (1990) elaboraram o Teste do Alcance Funcional (FRT). A análise dos resultados do teste evidencia que valores de 
deslocamento anterior inferiores a 15 centímetros $(\mathrm{cm})$ indicam fragilidade do paciente e risco de quedas (DUNCAN et al., 1990, BERG; MAKI; WILLIAMS, 1992, BERG et al., 1992, GOMES, 2003). Estudo de revisão sistemática de literatura evidenciou que o teste apresenta boa confiabilidade interexaminadores (SOARES et al., 2005).

Neste contexto, as alterações dos sistemas vestibulares e musculares têm grande importância, uma vez que o aumento da idade pode estar relacionado com distúrbios musculares, ou sintomas vestibulares, tais como as vertigens e as tonturas, ocorrendo um aumento no número de quedas em idosos, quando comparados com adultos (GANANÇA; MEZZALIRA; CRUZ, 2008, DUNCAN et al., 1990). Assim, este trabalho tem como objetivo verificar a existência de associação entre a queixa de tontura, os resultados do teste de alcance funcional e a ocorrência de quedas em idosos não institucionalizados.

\section{Metodologia}

A amostra deste estudo foi selecionada por conveniência, sendo composta por idosos não institucionalizados, residentes em uma cidade da região metropolitana de Porto Alegre, que compareceram à universidade para participar de atividades de extensão. Todos frequentavam grupos de terceira idade nos bairros onde residiam.

Foram incluídos nesta pesquisa indivíduos com idade igual, ou superior a 60 anos, residentes na cidade onde o projeto foi desenvolvido e participantes de grupos de terceira idade nos seus bairros.

Entre os critérios de exclusão estavam a institucionalização, a não aceitação em participar voluntariamente da pesquisa, pessoas com dificuldades de locomoção, portadores de morbidades nos membros inferiores e histórico de doenças neurológicas, cognitivas e psiquiátricas. Optou-se por excluir indivíduos com o histórico das doenças citadas em função da dificuldade de obtenção do Termo de Consentimento Livre e Esclarecido (TCLE) e da influência que as doenças citadas poderiam exercer nos resultados dos testes.

Inicialmente foram esclarecidos os objetivos e a metodologia da pesquisa e os indivíduos que aceitaram participar assinaram o TCLE. Após a assinatura, foram realizadas entrevistas individuais por acadêmicos e pesquisadores dos cursos de Graduação em Fonoaudiologia e Educação Física. Foi utilizado um questionário elaborado especificamente para este estudo, contendo questões que versavam sobre:

- dados sócio-demográficos (idade, sexo); 
- histórico auditivo e vestibular (presença/ausência de perda auditiva, tontura, realização de avaliações audiológicas e vestibulares, uso de aparelho de amplificação sonora individual);

- histórico de quedas nos últimos seis meses;

- cuidados para evitar futuras quedas.

A seguir, foi realizado o teste do alcance funcional anterior (DUNCAN et al., 1990). Tal como foi descrito na introdução, no teste do alcance funcional anterior o indivíduo é instruído a retirar os sapatos. A seguir ele deve posicionar-se perpendicularmente à parede, com os pés paralelos, sem apoiar-se contra a parede, com o braço fletido em $90^{\circ}$ e o cotovelo estendido. Marca-se a posição do terceiro metacarpo. A seguir, o indivíduo é orientado a deslocarse para frente, mantendo o braço estendido, sem retirar os calcanhares do chão e sem perder o equilíbrio. É feita uma nova medida, comparando-se o valor inicial e o valor final da posição do terceiro metacarpo. São feitas três medidas, sendo registrada a média dessas. Os idosos que alcançaram entre $15,2 \mathrm{~cm}$ e $25,4 \mathrm{~cm}$ de deslocamento apresentam um risco duas vezes maior de cair do que os idosos que alcançam mais do que $25,4 \mathrm{~cm}$. Já os idosos que atingiram menos que $15,2 \mathrm{~cm}$ têm quatro vezes mais chance de cair do que os idosos que atingem valores superiores a $25,4 \mathrm{~cm}$ de deslocamento.

Os dados foram analisados de forma estatística quantitativa, sendo analisada a associação entre as variáveis estudadas. A análise dos dados foi feita utilizando-se o programa SPSS versão 18, sendo considerado o nível de significância de $5 \%$. Para análise da associação entre queda e tontura foi utilizado o teste do Qui-Quadrado. Para verificar a existência de associação entre queda e os resultados do teste do alcance funcional foi utilizado o teste de Mann-Whitney, para amostras independentes. O teste de Kruskal-Wallis foi utilizado para avaliar a existência de associação entre tontura e os resultados do teste de alcance funcional.

\subsection{Aspectos éticos}

O projeto foi aprovado pelo Comitê de Ética em Pesquisa da instituição, sob protocolo número $166 \mathrm{H}$. Foram assegurados os direitos de sigilo, anonimato, desistência de participação e continuidade do atendimento prestado na universidade, caso os idosos não aceitassem participar da pesquisa, ou desistissem da mesma após a realização dos testes. 
A amostra foi composta por 50 idosos, sendo 43 (86\%) do sexo feminino. A idade dos entrevistados variou entre 60 e 83 anos (média de 69,96 anos \pm $5,80)$.

Com relação à queixa de tontura, 12 (24\%) sujeitos relataram que a sentiam de forma constante, 14 (28\%) às vezes e 24 (48\%) não apresentavam tal sintoma. Do total de idosos entrevistados, 12 (24\%) relatavam episódios de quedas nos seis meses anteriores a aplicação do questionário. Apesar disso, somente cinco $(10 \%)$ relataram ter adotado medidas de proteção para evitar quedas.

O teste do alcance funcional evidenciou que três idosos (6\%) atingiram índices inferiores a 15cm, 28 idosos (56\%), índices entre 15 e $25 \mathrm{~cm}$ e 19 idosos (38\%), índices superiores a $25 \mathrm{~cm}$. Segundo Duncan et al. (1990), idosos que alcançam até $15,2 \mathrm{~cm}$ têm quatro vezes mais chance de cair do que os idosos que alcançam mais de $25,4 \mathrm{~cm}$. Já os idosos que alcançam entre $15,2 \mathrm{~cm}$ e $25,4 \mathrm{~cm}$ têm duas vezes mais chance de cair do que idosos que alcançam mais do que $25,4 \mathrm{~cm}$.

Na Tabela 1 é apresentada a descrição dos resultados do teste do alcance funcional estratificado por queda e tontura.

Tabela 1 - Resultados do teste do alcance funcional estratificado por queda e tontura

\begin{tabular}{|c|c|c|c|c|c|}
\hline \multirow[b]{2}{*}{ Características } & \multirow[b]{2}{*}{ N } & \multicolumn{4}{|c|}{ Teste do alcance funcional - em centímetros } \\
\hline & & Média & $\begin{array}{l}\text { Desvio } \\
\text { Padrão }\end{array}$ & Mediana & $\begin{array}{l}\text { Amplitude } \\
\text { interquartil }\end{array}$ \\
\hline \multicolumn{6}{|l|}{ Queda } \\
\hline Não & 38 & 23,19 & 6,59 & 20,35 & 9,75 \\
\hline Sim & 12 & 26,50 & 5,41 & 26,00 & 8,63 \\
\hline \multicolumn{6}{|l|}{ Tontura } \\
\hline Não & 24 & 23,23 & 6,43 & 21,75 & 10,50 \\
\hline Sim & 12 & 22,71 & 6,15 & 20,00 & 9,00 \\
\hline Às vezes & 14 & 26,39 & 6,49 & 25,25 & 10,50 \\
\hline
\end{tabular}

Para observar a relação entre quedas e o resultado do teste do alcance funcional, foi utilizado o teste de Mann-Whitney para amostras independentes, pois o teste de Shapiro-Wilk rejeitou a hipótese de normalidade para a variável: alcance funcional. Observou-se que a média e a mediana do alcance 
funcional são maiores para o grupo de indivíduos que caiu, sugerindo que indivíduos que caíram possuem alcance funcional superior, em relação ao alcance funcional dos indivíduos que não caíram. Contudo, o valor de $\mathrm{p}=0,052$, não assegura, do ponto de vista estatístico, que essa relação é, de fato, observada na amostra em estudo. É provável que o tamanho da amostra tenha influenciado nos resultados. Talvez, com uma amostra maior fosse possível obter evidências para tal relação.

A análise estatística permitiu evidenciar que não houve associação entre a queixa de tontura e os resultados do teste do alcance funcional $(\mathrm{p}=0,220)$. Verificou-se que os indivíduos que relataram ter sintomas de tontura às vezes, tiveram resultados no teste do alcance funcional maiores do que os indivíduos que não declararam sintomas de tontura. Em se tratando dos grupos de indivíduos que afirmaram ter sintomas de tontura sempre, e que não declararam sintomas de tontura, as medidas de tendência central mostram-se bastante próximas. Assim, poderia se sugerir que os indivíduos com tonturas esporádicas teriam maior alcance funcional, em relação aos outros grupos (sim e não). Entretanto, a relação não foi significativa, evidenciando não haver relação entre a tontura e os resultados do teste do alcance funcional.

Na tabela 2 são apresentados os valores decorrentes do cruzamento das variáveis queixa de tontura e queda nos últimos seis meses.

Tabela 2 - Análise da associação entre as variáveis tontura e queda nos seis meses que antecederam a pesquisa

\begin{tabular}{cccccccc}
\hline & \multicolumn{3}{c}{ Quedas } & \multirow{2}{*}{ Total } \\
\cline { 3 - 6 } & & \multicolumn{3}{c}{ Não } & \multicolumn{3}{c}{$\operatorname{Sim}$} \\
\hline \multirow{2}{*}{ Tontura } & $\mathrm{n}$ & $\%$ & $\mathrm{n}$ & $\%$ & $\mathrm{n}$ & $\%$ \\
& Não & 21 & 55,33 & 3 & 25,0 & 24 & 48 \\
& Sim & 9 & 23,7 & 3 & 25,0 & 12 & 24,0 \\
& Às vezes & 8 & 21,1 & 6 & 50,0 & 14 & 28,0 \\
\hline Total & & 38 & 100 & 12 & 100 & 50 & 100 \\
\hline
\end{tabular}

A análise estatística evidenciou que não houve associação entre sintomas de tontura e a presença de queda $(p=0,107)$.

A prevalência de quedas observada na amostra foi semelhante ao relatado na literatura especializada. Segundo as pesquisas de Perracini e Ramos (2002), Liposcki e Rosa Neto (2008), aproximadamente 30\% dos idosos não institucionalizados sofrem uma queda ao ano. Essas quedas podem ser 
ocasionadas por distúrbios de equilíbrio, provocadas por alterações no funcionamento dos sistemas vestibular, proprioceptivo, ou visual (BOTTEGA; LOBO; TEIXEIRA, 2006) e também por problemas no ambiente onde o idoso reside (LOPES et al., 2007), entre outros fatores. Mesmo assim, tal como no trabalho de Lopes et al. (2007), somente uma pequena porcentagem dos idosos relatou tomar cuidados para evitar futuras quedas.

Resultados de uma pesquisa realizada no Reino Unido demonstraram que as quedas em idosos originam um custo equivalente a 3\% dos fundos do National Health Service (NHS), sendo que a maior parte dos idosos que caem tem sua qualidade de vida seriamente afetada (SNOOKS et al., 2010), pois as quedas provocam restrições na capacidade funcional. Destaca-se que esse tipo de restrição é preditor significativo de mortalidade, mas é um fator mutável (RAMOS, 2003), ou seja, com medidas adequadas, se poderia reduzir, não somente as quedas, mas também a incapacidade funcional e a mortalidade por esses fatores. Assim, a prevenção de quedas deveria ser uma prioridade, uma vez que programas podem ser criados e mantidos por profissionais das mais diversas especialidades (Fonoaudiologia, Educação Física, Fisioterapia, Medicina, por exemplo), visando não só uma redução de gastos públicos, mas especialmente a manutenção da qualidade de vida dos idosos, que poderiam manter-se ativos por mais tempo, evitando-se todas as consequências negativas das quedas.

A não associação entre a presença de tontura e histórico de quedas nos seis meses que antecederam a entrevista corrobora dos achados de Gai et al. (2010), evidenciando que, na amostra estudada, a presença desse sintoma não foi um fator determinante para a perda de equilíbrio. Já a ausência de associação entre o teste do alcance funcional anterior (DUNCAN et al., 1990) e o histórico de quedas, diferiram dos resultados obtidos por Gai et al. (2010). Destaca-se que tais resultados também contrariam a hipótese do estudo, uma vez que, pelas informações obtidas na literatura e pela experiência dos pesquisadores, esperava-se que houvesse associação entre tontura e histórico de quedas e entre os resultados do teste do alcance funcional e o histórico de quedas (DUNCAN et al., 1990; TINETTI et al., 2000; GAZOLLA et al., 2006). O tamanho da amostra pode ter sido um fator influenciador negativo, não possibilitando encontrar resultados significativos na análise estatística.

Os resultados obtidos talvez possam ser explicados pelo caráter multidimensional das quedas, ou seja, não é somente um fator isolado que leva o idoso a cair, mas um conjunto de determinantes físicos, ambientais e emocionais. Os dados confirmam a importância da atuação interdisciplinar na prevenção de quedas. Profissionais que atuam com atenção primária e 
programas de saúde da família deveriam incluir orientações sobre noções de risco e consequências das quedas, bem como, identificar idosos de risco.

A reabilitação de distúrbios visuais, de equilíbrio, marcha e força deveria constar como prioridade, buscando a manutenção do idoso ativo na comunidade (MOTTA et al., 2010). Além disso, a abordagem reabilitativa, com o idoso que já sofreu uma, ou várias quedas também deveria ser interdisciplinar, uma vez que somente o treinamento de força, ou flexibilidade pode não levar ao resultado esperado. Muitas vezes é necessária a reabilitação vestibular, com exercícios escolhidos de forma personalizada (PATATAS; GANANÇA; GANANÇA, 2009). Isso facilitaria, não só a retomada do equilíbrio corporal, mas também a manutenção das atividades de vida diária e profissional, minimizando os efeitos emocionais e psicológicos da tontura, melhorando, assim, a qualidade de vida dos idosos (MANTELLO et al., 2008, GAZOLLA et al., 2009).

Este estudo será continuado, visando ampliar o tamanho da amostra e descrever com mais especificidade a situação das quedas sofridas pelos idosos, bem como estabelecendo programas de intervenção para a prevenção das mesmas.

4 Conclusão

Os resultados deste estudo evidenciam que não houve associação entre quedas, presença de tontura e resultados do teste do alcance funcional entre os idosos avaliados.

DIZZINESS, FALLS AND FUNCTIONAL
REACH TEST: RESULTS OBTAINED
IN NON-INSTITUTIONALIZED ELDERLY

abstract

This study aimed to investigate the association among dizziness, functional reach test and history of falls. Fifty patients were evaluated, using interviews prepared for this study and the functional reach test. Results showed that $52 \%$ of elderly had dizziness and $24 \%$ reported a fall in the last 6 months. In the functional reach test, results pointed that 38\% of the elderly reached values higher than $25 \mathrm{~cm}$. 

Whitney and Kruskal-Wallis tests and demonstrated that there was no association between the variables studied.

keywords

Dizziness. Falls. Elderly. Postural Balance.

\author{
referências
}

BERG K.O.; MAKI B.; WILLIAMS, J. Clinical and laboratory measures of postural balance in an elderly population. Archives of Physical Medicine Rehabilitation, Philadelphia, v. 73, p. 1073-80,1992.

BERG, K.O et al. Measuring balance in the elderly: validation of an instrument. Canadian Journal of Public Health, Otawa, v. 83, Suppl 2, S7-S11, 1992.

BOTTEGA, Fabiane; LOBO, Marilda Biacchi; TEIXEIRA, Adriane Ribeiro. "Performanceoriented mobility assessment of gait and balance - Brasil" (POMA Brasil): um instrumento auxiliar na avaliação e diagnóstico otoneurológico do paciente idoso. Revista de Iniciação Científica da ULBRA, Canoas, v. 5, p. 87-98, 2006

BRONSTEIN, Adolfo, LEMPERT, Thomas. Tonturas - Diagnóstico e tratamento - uma abordagem prática. Rio de Janeiro: Revinter, 2010.

DUNCAN, Pamela W et al. Stephanie. Functional reach: A new clinical measure of balance. Journal of Gerontology, Cary, v. 45, n. 6, p. M192-197, 1990.

FABRÍCIO, Suzele Cristina Coelho; RODRIGUES, Rosalina A. Partezani; COSTA Jr, Moacyr Lobo da. Causas e conseqüências de quedas de idosos atendidos em hospital público. Revista de Saúde Pública, São Paulo, v. 38, n. 1, p. 93-99, fev. 2004.

FANELLI, Rosamaria; BARRETO FILHO, Antonio; RAQUEL, Sandra. Avaliação multidisciplinar em idosos com tontura. ConScientiae Saúde, São Paulo, v. 1, p. 31-33. 2002.

FELIPE, Lilian et al. Presbivertigem como causa de tontura no idoso. Pró-Fono Revista de Atualização Científica, Barueri, v. 20, n. 2, p. 99-104, abr./jun. 2008.

GAl, Juliana et al. Fatores associados a quedas em mulheres idosas residentes na comunidade. Revista da Associação Médica Brasileira, São Paulo, v. 56, n. 3, p. 327 332. 2010.

GANANÇA, Maurício Malavasi; CAOVILLA, Heloisa Helena. Como lidar com as tonturas e sintomas associados. In: GANANÇA, Mauricio Malavasi et al. Estratégias terapêuticas em otoneurologia. São Paulo: Atheneu, 2000. p. 1-20.

GANANÇA, Fernando; MEZZALIRA, Raquel; CRUZ, Osvaldo Laércio. Campanha Nacional de prevenção a quedas de idosos. Revista Brasilleira de Otorninolaningologia, São Paulo, v. 74, n. 2, p. 162, mar./abr. 2008

GAZZOLA, Juliana Maria et al. Circunstâncias e conseqüências de quedas em idosos com vestibulopatia crônica. Revista Brasileira de Otorrinolaringologia, São Paulo, v. 72, n. 3, p. 388-393, maio/jun. 2006.

GAZZOLA, Juliana Maria et al. Factors relating depressing symptoms among elderly people with chronic vestibular diysfunction. Arquivos de Neuro-psiquiatria, São Paulo, v. 67, n. 2b, p. 416-422, jun. 2009. 
GOMES, Gisele de Cássia. Tradução, adaptação cultural e exame das propriedades de medida da escala "Performance-oriented mobility assessment" (POMA) para uma amostragem de idosos brasileiros institucionalizados. 110f. Dissertação (Mestrado em Gerontologia) - Programa de Pós Graduação em Educação, Universidade Estadual de Campinas, Campinas, 2003.

GUIMARÃES, L.H.C.T. et al. Comparação da propensão de quedas entre idosos que praticam atividade física e idosos sedentários. Neurociências, São Paulo, v. 12, n. 2, abr-jun. 2004. Disponível em <http://www.unifesp.br/dneuro/neurociencias/vol12_2/ quedas.htm>. Acesso em: 27 de ago. 2010.

GUIMARÃES, Joana Miguez Nery; FARINATTI, Paulo de Tarso Veras. Análise descritiva de variáveis teoricamente associadas ao risco de quedas em mulheres idosas. Revista Brasileira de Medicina do Esporte, São Paulo, v. 11, n. 5, p. 299-305, set./out. 2005.

KOGA, Katia Akemi; RESENDE, Bruna D'Arce; MOR, Rita. Estudo da prevalência de tontura/vertigens e das alterações vestibulares relacionadas à mudança na posição da cabeça por meio da vectoeletronistagmografia computadorizada. Revista CEFAC, São Paulo, v. 6, n. 2, p. 197-202, abr./jun. 2004.

LIPOSCKI, Daniela Branco; ROSA NETO, Francisco. Prevalência de artrose, quedas e a relação com o equilíbrio dos idosos. Terapia Manual, São Paulo, v. 6, n. 26, p. 235 238, jul./ago. 2008.

LOPES, Mislaine de Lima et al. Fatores desencadeantes de quedas no domicílio em uma comunidade de idosos. Cogitare Enfermagem, Curitiba, v. 12, n.4, p. 472-477, out./dez. 2007.

LOPES, Renata Antunes et al. Quedas de idosos em uma clínica-escola: prevalência e fatores associados. ConScientiae Saude, São Paulo, v. 9, n. 3, p. 381-388. 2010.

MACHADO, Tatiana Rocha et al. Avaliação da presença de risco para quedas em idosos. Revista Eletrônica de Enfemagem, Goiânia, v. 11, n. 1, p. 32-38. 2009. Disponível em: <http://muw.gen.ufg.br/revista/v11/n1/v11n1a04.htm>. Acesso em: 26 de ago. 2010 .

MANTELLO, Erika Barioni et al. Efeito da reabilitação vestibular sobre a qualidade de vida de idosos labirintopatas. Revista Brasileira de Otominolaringologia, São Paulo, v. 74, n. 2 , p. 172-180, mar./abr. 2008.

MOTTA, Luciana Luciana Branco et al. Prevalência e fatores associados a quedas em idosos de um município do Rio de Janeiro. Revista Brasilleira de Geriatria e Gerontologia, Rio de Janeiro, v. 13, n. 1, p. 83-92, 2010.

PATATAS, Olívia Helena Gomes; GANANÇA, Cristina Freitas; GANANÇA, Fernando Freitas. Qualidade de vida de indivíduos submetidos a reabilitação vestibular. Brazilian Joumal of Otorhinolaryngology, São Paulo, v. 75, n. 3, p. 387-394, mai./jun. 2009.

SOCIEDADE BRASILEIRA DE GERIATRIA E GERONTOLOGIA. Quedas em idosos: prevenção. Projeto Diretrizes AMB e CFM. 2008. Disponível em: <http://unw. projetodiretrizes.org.br/projeto_diretrizes/082.pdf>. Acesso em: 13 de set. 2011.

PERRACINI, Monica Rodrigues; RAMOS, Luiz Roberto. Fatores associados a quedas em uma coorte de idosos residentes na comunidade. Revista de Saúde Pública, São Paulo, v. 36, n. 6, p. 709-716. 2002.

RAMOS, Luiz Roberto. Fatores determinantes do envelhecimento saudável em idosos residentes em centro urbano: Projeto Epidoso. São Paulo, Cademos de Saúde Pública, Rio de Janeiro, v. 19, n. 3, p. 793-797, mai./jun. 2003.

SOARES, Karla Vanessa et al. Avaliação quanto a utilização e confiabilidade de instrumentos de medida do equilíbrio corporal em idosos. PublilCa, Natal, v. 1, p. 78-85, 2005. 
Recebido: 23/12/2010

1a Revisão: 19/07/2011

2a Revisão: 17/08/2011

Aceite Final: 02/09/2011
SNOOKS, Helen et al. Support and Assessment for Fall Emergency Referrals (SAFER 1) trial protocol. Computerised on-scene decision support for emergency ambulance staff to assess and plan care for older people who have fallen: evaluation of costs and benefits using a pragmatic cluster randomised trial. BMC Emerg, Londres, v. 10, n. 2. 2010. Disponível em: <http://wnw.ncbi.nlm.nih.gov/pmc/articles/ PMC2824628/?tool=pubmed>. Acesso em: 04 de dez. 2010.

TINEITI, Mary et al. Dizziness among older adults: a possible geriatric syndrome. Annals of intemal Medicine, Philadelphia, v. 132, n. 5, p. 337-344, 2000. 